\title{
SCIM - spinal cord independence measure: a new disability scale for patients with spinal cord lesions
}

\author{
A Catz ${ }^{1}$, M Itzkovich ${ }^{1}$, E Agranov $^{1}, \mathrm{H} \mathrm{Ring}^{1}$ and A Tamir ${ }^{2}$ \\ ${ }^{1}$ Loewenstein Rehabilitation Hospital, Raanana, and Sackler Faculty of Medicine, Tel Aviv University, Tel Aviv, \\ Israel; ${ }^{2}$ Department of Community Medicine, Faculty of Medicine, Technion, Haifa, Israel
}

\begin{abstract}
The Spinal Cord Independence Measure (SCIM) is a new disability scale developed specifically for patients with spinal cord lesions in order to make the functional assessments of patients with paraplegia or tetraplegia more sensitive to changes. The SCIM includes the following areas of function: self-care (subscore $0-20)$, respiration and sphincter management $(0-40)$ and mobility $(0-40)$. Each area is scored according to its proportional weight in these patients' general activity. The final score ranges from 0 to 100 . This study was performed to evaluate the reliability of the SCIM and its sensitivity to functional changes in spinal cord lesion patients compared with the Functional Independence Measure (FIM). Thirty patients were included. Scores were recorded one week after admission and thereafter every month during hospitalization. Each area of function was assessed by a pair of staff members from the relevant discipline. The comparison of scores between each pair of raters revealed a remarkable consistency $(r=0.91-0.99 ; P<0.0001 ;$ slope $\approx 1 ;$ constant $\approx 0)$. The total SCIM score $($ mean $=51, \mathrm{SD}=21)$ was lower than the total FIM score $($ mean $=87, \mathrm{SD}=23)$ owing to the difference in scale range structure and the relatively high cognitive scores of our patients; however, a relationship was noted between the scores of both scales $(r=0.85, P<0.01)$. The SCIM was more sensitive than the FIM to changes in function of spinal cord lesion patients: the SCIM detected all the functional changes detected by the FIM total scoring, but the FIM missed $26 \%$ of the changes detected by the SCIM total scoring. The mean difference between consecutive scores was higher for the SCIM $(P<0.01)$. We conclude that the SCIM is a reliable disability scale and is more sensitive to changes in function in spinal cord lesion patients than the FIM. The SCIM when administered by a multidisciplinary team, may be a useful instrument for assessing changes in everyday performance in patients with spinal cord lesion.
\end{abstract}

Keywords: SCIM; spinal cord; ADL; disability; functional assessment; validity; reliability

\section{Introduction}

Standardized rating scales are a customary measure in rehabilitation medicine for the functional assessment of patients with various disabilities. Among the objectives of these scales are: unbiased quantitative evaluation of patient function, assessment of treatment efficacy, and estimation of assistance necessary. ${ }^{1,2}$ The three scales most often used in patients with spinal cord lesion (SCL) are: the Modified Barthel Index (MBI), ${ }^{3}$ the Functional Independence Measure (FIM), ${ }^{4}$ and the Quadriplegia Index of Function (QIF). ${ }^{5}$ Several authors have found the MBI and the FIM appropriate for SCL patients, ${ }^{1,2,6,7}$ although others, such as Gresham et $a l^{5}$ and Marino et $a l^{8}$ raised doubts about their efficiency in measuring functional changes in this population. We, too, believe the MBI and the FIM,

Correspondence: A Catz which were developed for the functional assessment of patients with several different kinds of impairments, do not satisfactorily reflect the rehabilitation outcome in SCL patients. Our impression is that they lack sensitivity to functional changes and do not attach sufficient importance to certain achievements of these patients. The QIF has overcome some of the limitations of the MBI and FIM, but it was designed especially for patients with tetraplegia and is not suitable for the assessment of patients with paraplegia. For example, it does not include an evaluation of mobility functions.

To fill this gap, a new scale for the functional assessment of patients with SCL was developed at the Department of Spinal Rehabilitation of Loewenstein Rehabilitation Hospital. This scale, the Spinal Cord Independence Measure (SCIM) (Appendix A), has the following advantages: (a) Areas of function relevant to SCL patients are included, and abilities that are often not so disturbed as to affect basic everyday functioning, such as cognitive abilities, are excluded; (b) 
Scoring is relatively high for those achievements we consider more important in SCL patients; (c) Each area of function is assessed according to its weight relative to the overall activity, as determined specifically for this population; (d) Scoring criteria are precisely defined and presented in the evaluation sheet.

The SCIM covers three principal areas of function: self-care, respiration and sphincter management, and mobility (Appendix A). Self-care includes the following tasks: feeding, bathing, dressing and grooming; scores for this area range from 0 to 20. Respiration and sphincter management includes: respiration, bladder management, bowel management and use of toilet; scores for this area range from 0 to 40 . Mobility is divided into two parts: tasks performed in the room and toilet, and tasks performed all over the house (indoors) and outdoors. Mobility in room and toilet includes: mobility in bed and action to prevent pressure sores, and transfers of bed-wheelchair and wheelchairtoilet-tub. Mobility indoors and outdoors includes: mobility for short, moderate and long distances, stair management and transfers of wheelchair-car. Scores for this area range from 0 to 40 . The purpose of the study was to examine the reliability and sensitivity of the SCIM to functional changes in SCL patients. The study hypotheses were: (1) Functional assessment by the SCIM shows a high interrater reliability; (2) The SCIM is more sensitive than the FIM to functional changes in patients with SCL.

\section{Patients and methods}

Thirty patients (22 males, 8 females) admitted to the Department of Spinal Rehabilitation of Loewenstein Rehabilitation Hospital from December 1994 to October 1995 were included in the study. Since SCIM was intended to test the effect of SCL on function, patients with concomitant impairments such as brain injury or mental disease that might influence their everyday function were excluded. Ages ranged between 17 and 76 years $($ mean $=45$, s.d. $=18)$. Nine patients had tetraplegia and 21 had paraplegia. In 9 patients the lesions were complete or almost complete on admission (Frankel A or B), and in 21 they were incomplete (Frankel C or D). Fifteen of the spinal lesions were traumatic; the remainder were compressive degenerative spine lesions, meningioma and achondroplasia. All patients were evaluated with the SCIM and the FIM for the first time one week after admission to the department and thereafter every month during hospitalization. Follow-up ranged from one to six months (three months on average). The time needed for each evaluation with either the SCIM or the FIM was 30 to $45 \mathrm{~min}$. Each area of function on the SCIM was scored by two staff members in the relevant field, as follows: self-care - occupational therapists; respiration and sphincter management and mobility in the room and toilet - nurses; mobility indoors and outdoors - physiotherapists. Each of the examiners scored the patients independently and was blind to the other examiners' results. To determine interrater reliability the relationship between the SCIM scores obtained by the pairs of staff members was evaluated with three methods: (a) percentage of examinations in which the scoring of the two raters was identical (total agreement); (b) chance-corrected measure of agreement (Kappa) ${ }^{9}$ (c) linear regression and Pearson correlation coefficient. The analysis included examinations performed by two paired raters within 1 week.

To determine relative sensitivity of the test to functional changes, fluctuations in the scores on the SCIM (measured by the staff) and on the FIM (measured by a nurse) were compared throughout the rehabilitation period for: (a) comparing the rate of detection of functional changes, by McNemar test ${ }^{9}$ (b) mean differences between last and first scores and between all consecutive scores, by paired $t$-test. As the range of scores is $1-100$ for the SCIM and 18-126 for the FIM, FIM scoring, F, was normalized to $\mathrm{F}^{*}$ using the formula: $\mathrm{F}^{*}=(\mathrm{F}-18) /(126-18) \times 100$

The relationship between the total scores on the SCIM and the FIM was tested by Pearson correlation coefficient.

The content and construct of the SCIM were discussed with the spinal department's multidisciplinary team before its composition and following its examination. The conclusions of these discussions contributed to the evaluation of SCIM's validity. Data were analyzed with the SPSS.

\section{Results}

\section{Interrater reliability}

Results showed a very high agreement between raters and high reliability of the SCIM. Total interrater agreement on the various individual tasks ranged between 72 and $99 \%$; for most of the SCIM tasks, total agreement was higher than $85 \%$, and the Kappa coefficient ranged between 0.66 and 0.98 . Relatively high values of total agreement were obtained in tasks of mobility, and relatively low values in tasks of motion in bed, sphincter management, and dressing (Table 1). Subscores obtained by the pairs of raters in the different areas of function were highly correlated, with correlation coefficients ranging between 0.91 and $0.99(P<0.001)$. The slope values of the regression lines of the scores of paired raters were very close to 1 , and they crossed the axes very close to 0 . These findings indicate that the scores of different raters were similar in the various areas of function (Table 2). Very high correlations and similar coefficients of the linear regression were also found between the total SCIM scores of the paired teams $(r=0.98, \quad P<0.001)$ (Figure 1).

\section{Sensitivity to functional changes}

The SCIM was found to be more sensitive than the FIM to changes in function in SCL patients. The 
SCIM detected all the functional changes detected by FIM total scoring, but in $15(26 \%)$ of 57 sequential test batteries, the FIM missed changes detected by SCIM total scoring. In 40 of the 57 examinations $(70.1 \%)$, the functional changes were detected by both scales. These differences between the scales were significant $(P<0.001)$. Fifty-one of the 55 functional changes detected by at least one scale, and 14 of the 15 functional changes detected by the SCIM, were improvements. When the change in the individual areas of function was examined, the detection rate was significantly higher for the SCIM than for the FIM for respiration/sphincter management and mobility indoors and outdoors $(P<0.001)$; no significant differences were found for the other two areas.

Consecutive scores on the SCIM showed a significantly higher mean difference than those on the FIM $\left(\mathrm{F}^{*}\right)(10.6$ vs $7.5 ; t=2.7, P<0.01)$. When the differences between the last and first total scores were compared, the mean difference was also significantly

Table 1 Agreement between raters on the various individual tasks:SCIM

\begin{tabular}{|c|c|c|c|}
\hline Task & $\mathrm{n}$ & $\begin{array}{c}\text { Total } \\
\text { agreement }\end{array}$ & $\begin{array}{l}\text { Kappa } \\
\text { coefficient }\end{array}$ \\
\hline Self-care & 92 & & \\
\hline Feeding & & $86.9 \%$ & 0.696 \\
\hline Bathing & & $79.3 \%$ & 0.730 \\
\hline Dressing & & $75.0 \%$ & 0.696 \\
\hline Grooming & & $85.8 \%$ & 0.733 \\
\hline Respiration and sphincter & 67 & & \\
\hline \multicolumn{4}{|l|}{ management } \\
\hline Respiration & & $95.5 \%$ & 0.711 \\
\hline Sphincter management-bladder & & $89.5 \%$ & 0.826 \\
\hline Sphincter management-bowel & & $77.6 \%$ & 0.657 \\
\hline Use of toilet & & $82.0 \%$ & 0.747 \\
\hline Mobility in room and toilet & 67 & & \\
\hline $\begin{array}{l}\text { Motion in bed and sore } \\
\text { prevention }\end{array}$ & & $71.6 \%$ & 0.656 \\
\hline Transfers: bed-wheelchair & & $88.0 \%$ & 0.807 \\
\hline $\begin{array}{l}\text { Transfers: wheelchair-toilet-tub } \\
\text { Mobility indoors and outdoors }\end{array}$ & 82 & $88.0 \%$ & 0.762 \\
\hline Mobility indoors & & $95.1 \%$ & 0.937 \\
\hline Mobility for moderate distances & & $96.2 \%$ & 0.947 \\
\hline Mobility outdoors & & $98.7 \%$ & 0.983 \\
\hline Stair management & & $92.6 \%$ & 0.840 \\
\hline Transfers: wheelchair-car & & $97.5 \%$ & 0.944 \\
\hline
\end{tabular}

$n=$ Number of tests included in the analysis higher for the SCIM than for the FIM $\left(\mathrm{F}^{*}\right)$ (25.9 vs $19.3 ; t=2.5, P<0.02)$.

Relationship between SCIM and FIM total scores

The mean total SCIM score was 51.2 (s.d.=21), and the mean total FIM score $(\mathrm{F})$ was 86.8 (s.d.=23). A positive correlation was found between the two scales $(r=0.85, P<0.01)$.

\section{Comments of the multidisciplinary team members,} including the raters

All the multidisciplinary team members felt that the SCIM scoring was a reliable reflection of the functional status of the SCL patients and the changes in functional status during rehabilitation. The raters noted that functional assessment by the SCIM was convenient owing to the inclusion of the scoring criteria on the evaluation sheet. However, the raters pointed out several drawbacks: (a) In self-care, the scoring for bathing and dressing was misleading when the main disability was in the upper part of the body; (b) In sphincter management, the need for assistance in applying an external urinary device or for undressing the lower body before otherwise independent use of the toilet was not covered by the scoring criteria; (c) In the area of mobility in room and toilet, the score for push-

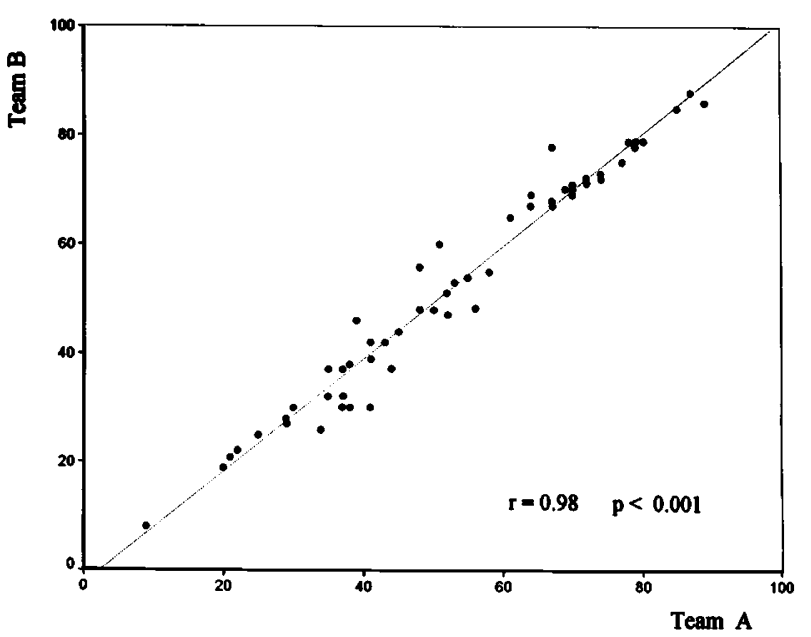

Figure 1 Correlation between total SCIM scores of two teams of raters

Table 2 Correlation between raters in the various areas of function

\begin{tabular}{llrrr}
\hline Raters & $N$ & $r$ & \multicolumn{1}{c}{$A$} & $B$ \\
\hline Occupational therapists (self-care) & 92 & 0.983 & -0.378 & 1.005 \\
Nurses (respiration and sphincter management) & 67 & 0.966 & -0.875 & 1.036 \\
Nurses (mobility in room and toilet) & 67 & 0.910 & 0.242 & 0.919 \\
Physiotherapists (mobility indoors and outdoors) & 82 & 0.992 & -0.112 & 0.997 \\
\hline
\end{tabular}

$\mathrm{N}=$ Number of tests included in the analysis. $\mathrm{A}=$ Intercept of the regression line and the vertical axis. $\mathrm{B}=$ Slope of the regression line 
ups in the sitting position only was exaggerated; (d) In the area of mobility indoors and outdoors, the need for supervision during mobility was not properly reflected, and transfer from wheelchair to car using adaptive devices should have been scored separately from assisted transfer.

\section{Discussion}

The results of this study support the two hypotheses: the SCIM is reliable and the SCIM is more sensitive than the FIM to functional changes in SCL patients. The statistical significance of the results of the different analyses indicates that the new scale is a good measure of the efficiency of rehabilitation treatment in SCL patients.

The scale was developed to fill the need for a rating instrument that measures and reflects the meaningful functional changes in SCL patients. At present, no scales are available to provide answers to the specific functional problems of this population. Marino et $a l^{8}$ pointed out that if a scale is not sensitive enough, it will miss real changes in patients' functional status. Law and Letts ${ }^{10}$ claimed that responsiveness to changes is the most important evidence of the validity of measures of activity of daily living (ADL). Accordingly, the SCIM's sensitivity to functional changes supports its validity.

Other aspects of the SCIM's validity are supported by the reports of the multidisciplinary team members and the quantitative findings of this study. Content and face validity are supported by the team's impression that the SCIM includes all areas of function that are relevant to the treatment goals in SCL patients and by the comments made by the raters. Construct (convergent) validity is supported by the correlation we found between the total scores on the SCIM and on the FIM, in view of earlier demonstrations of the validity of the FIM in SCL patients. ${ }^{7,11}$

Concurrent criterion-related validity is difficult to establish, as none of the existing scales can serve as a 'gold-standard' because of the limitations mentioned before. Future studies are planned to establish the predictive criterion-related validity of the SCIM in order to determine its ability to foresee rehabilitation outcome.

The main difference between the SCIM and the FIM is the relative weight given to the different everyday tasks. The main disabilities affecting everyday function in SCL patients are poor sphincter control and poor mobility. Rehabilitation treatment is largely focused on these areas, and success in dealing with these problems contributes to both life expectancy and quality of life. Therefore, the relative weight given to these areas is greater in the SCIM than in the FIM, as are the detailed criteria for their scoring. In the mobility subscale, for example, patients are scored not only according to the support they need, but also according to the distances they can achieve (Appendix A).
Cognitive, linguistic and psychosocial functions may be very important for rehabilitation outcome in SCL patients, but in our opinion these should be evaluated separately from everyday activities. This view is supported by Davidoff et al. ${ }^{12}$ Their findings indicate that cognitive evaluation as a part of disability assessment in SCL patients adds little to the information obtained and may decrease the sensitivity of the scale to changes in everyday performance.

The presentation of the detailed scoring criteria in the evaluation sheet makes the SCIM user-friendly and decreases the need to consult a manual, which raters find inconvenient. In spite of the generally encouraging results, in some of the SCIM tasks interrater reliability was not so high, and the differences in the scores for functional changes between the FIM and the SCIM were not significant. Therefore, the scoring criteria for these tasks probably need to be rephrased.

\section{Conclusion}

The SCIM shows a high interrater reliability, is more sensitive than the FIM to changes in function of SCL patients, rates functional achievements according to their importance for these specific patients (as based on our experience), includes ADL functions relevant to SCL patients, and defines scoring criteria on the evaluation sheet. Even though a few of the scoring criteria need to be rephrased, the SCIM, when administered by a multidisciplinary team is a useful instrument for assessing everyday performance in patients with spinal cord lesion.

\section{Acknowledgements}

This study was supported by the Unit of Medical Services, Rehabilitation Department, Ministry of Defence (Grant 11006-94).

\section{References}

1 Roth E, Davidoff G, Haughton J, Ardner M. Functional assessment in spinal cord injury: a comparison of the Modified Barthel Index and the 'adapted' Functional Independence Measure. Clinical Rehabilitation 1990; 4: $277-285$.

2 Grey N, Kennedy P. The Functional Independence Measure: a comparative study of clinician and self ratings. Paraplegia 1993; 31: $457-461$.

3 McDowell I, Neweell C. Measuring Health: a guide to rating scales and questionnaires. Oxford University Press 1987, pp. 49-54.

4 Uniform Data System for the Medical Rehabilitation and the Center for Functional Assessment Research. SUNY Buffalo, 82 Fuher Hall: SUNY Main St. Buffalo, NY 14214.

5 Gresham GE et al. The Quadriplegic Index of Function (QIF): sensitivity and reliability demonstrated in a study of thirty quadriplegic patients. Paraplegia 1986; 24: $38-44$.

6 Lammertse DP, Yarkony GM. Rehabilitation in spinal cord disorders: Outcomes and issues of aging after spinal cord injury. Archives of Physical Medicine and Rehabilitation 1991; 72: $309-$ 311.

7 Segal ME, Ditunno JF, Staas WE. Interinstitutional agreement of individual Functional Independence Measure (FIM) items measured at two sites on one sample of SCI patients. Paraplegia 1993; 31: $622-631$ 
8 Marino RJ et al. Assessing selfcare status in quadriplegia: comparison of the Quadriplegia Index of Function (QIF) and the Functional Independence Measure (FIM). Paraplegia 1993; 31: $225-233$

9 Fleiss JL. Stasticial Methods for Rates and Proportions. John Wiley \& Sons, 1981.

10 Law M, Letts L. A critical review of scales of activities of daily living. American Journal of Occuptational Therapy 1989; 43: $522-527$
11 Dodds TA, Martin DP, Stover WC, Deyo RA. A validation of the Functional Independence Measurement and its performance among rehabilitation inpatients. Archives of Physical Medicine and Rehabilitation 1993; 74: 531 - 560.

12 Davidoff GN, Roth EJ, Haughton JS and Ardner MS. Cognitive dysfunction in spinal cord injury patients: sensitivity of the Functional Independence Measure subscales vs neuropsychologic assessment. Archives of Physical Medicine and Rehabilitation 1990; 71: $326-329$

\section{Appendix A. Part 1}

\section{SCIM - SPINAL CORD INDEPENDENCE MEASURE Loewenstein Rehabilitation Hospital, Department IV (Version1, May 1996, Raanana, Israel)
Patient Name:
ID
Examiner Name:

(The score attached to the relevant description of each function should be placed in the adjacent square below the relevant date)

\section{Self-Care}

1. Feeding (cutting, opening containers, bringing food to mouth, holding cup with fluid)

0 . Needs parenteral, gastrostomy or fully assisted oral feeding

1. Eats cut food using several adaptive devices for hand and dishes

2. Eats cut food using only one adaptive device for hand; unable to hold cup

3. Eats cut food with one adaptive device; holds cup

4. Eats cut food without adaptive devices; needs a little assistance (e.g., to open containers)

5. Independent in all tasks without any adaptive device

\section{Bathing (soaping, manipulating water tap, washing)}

0 . Requires total assistance

1. Soaps only small part of body with or without adaptive devices

2. Soaps with adaptive devices; cannot reach distant parts of the body or cannot operate a tap

3. Soaps without adaptive devices; needs a little assistance to reach distant parts of body

4. Washes independently with adaptive devices or in specific environmental setting

5. Washes independently without adaptive devices

3. Dressing (preparing clothes, dressing upper and lower body, undressing)

0 . Requires total assistance

1. Dresses upper body partially (e.g., without buttoning) in special setting (e.g., back support)

2. Independent in dressing and undressing upper body. Needs much assistance for lower body

3. Requires little assistance in dressing upper or lower body

4. Dresses and undresses independently, but requires adaptive devices and/or special setting

5. Dresses and undresses independently, without adaptive devices

\section{Grooming (washing hands and face, brushing teeth,} combing hair, shaving, applying makeup)

0 . Requires total assistance

1. Performs only one task (e.g., washing hands and face)

2. Performs some tasks using adaptive devices; needs help to put on/take off devices

3. Performs some tasks using adaptive devices; puts on/takes off devices independently

4. Performs all tasks with adaptive devices or most tasks without devices

5. Independent in all tasks without adaptive devices
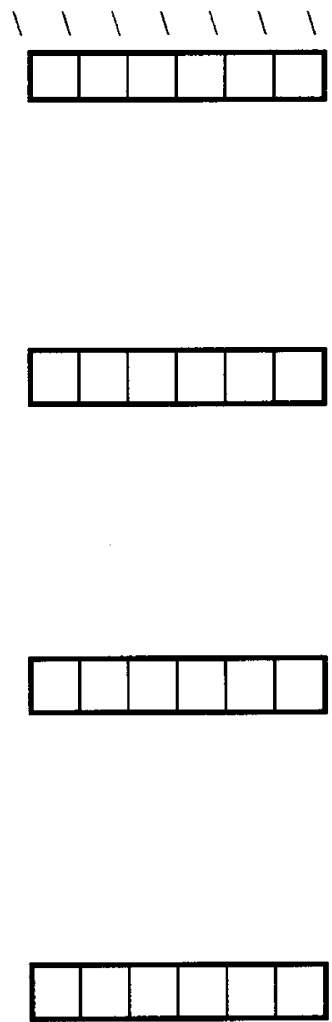


\section{Appendix A. Part 2}

\section{Respiration and Sphincter Management}

DATE

5. Respiration

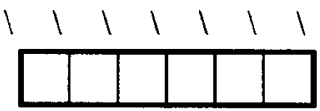

0 . Requires assisted ventilation

2. Requires tracheal tube and partially assisted ventilation

4. Breathes independently but requires much assistance in tracheal tube management

6. Breathes independently and requires little assistance in tracheal tube management

8. Breathes without tracheal tube, but sometimes requires mechanical assistance for breathing

10. Breathes independently without any device

\section{Sphincter management - Bladder}

0 . Indwelling catheter

5. Assisted intermittent catheterization or no catheterization, residual urine volume $>100 \mathrm{cc}$

10. Intermittent self-catheterization

15. No catheterization required, residual urine volume $<100 \mathrm{cc}$

\section{Sphincter management - Bowel}

0 . Irregularity, improper timing or very low frequency (less than once in 3 days) of bowel movements

5. Regular bowel movements, with proper timing, but with assistance (e.g., for applying suppository)

10. Regular bowel movements, with proper timing, without assistance

8. Use of toilet (perineal hygiene, clothes adjustment before/after, use of napkins or diapers)

0 . Requires total assistance

1. Undresses lower body, needs assistance in all the remaining tasks

2. Undresses lower body and partially cleans self (after); needs assistance in adjusting clothes and/or diapers

3. Undresses and cleans self (after); needs assistance in adjusting clothes and/or diapers

4. Independent in all tasks but needs adaptive devices or special setting (e.g., grab-bars)

5. Independent without adaptive devices or special setting

\section{Mobility (room and toilet)}

\section{Mobility in bed and action to prevent pressure sores}

0 . Requires total assistance

1. Partial mobility (turns in bed to one side only)

2. Turns to both sides in bed but does not fully release pressure

3. Releases pressure when lying only

4. Turns in bed and sits up without assistance

5. Independent in bed mobility; performs push-ups in sitting position without full body elevation

6. Performs push-ups in sitting position

10. Transfers: bed-wheelchair (locking wheelchair, lifting footrests, removing and adjusting arm rests, transferring, lifting feet)

0 . Requires total assistance

1. Needs partial assistance and/or supervision

2. Independent

11. Transfers: wheelchair-toilet-tub (if uses toilet wheelchair - transfers to and from; if uses regular wheelchair - locking wheelchair, lifting footrests, removing and adjusting

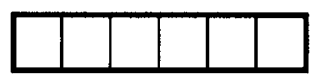
arm rests, transferring, lifting feet)

0 . Requires total assistance

1. Needs partial assistance and/or supervision, or adaptive device (e.g., grab-bars)

2. Independent 


\section{Appendix A. Part 3}

\section{Mobility (indoors and outdoors)}

12. Mobility indoors (short distances)

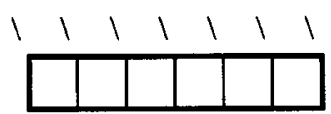

0 . Requires total assistance

1. Needs electric wheelchair or partial assistance to operate manual wheelchair

2. Moves independently in manual wheelchair

3. Walks with a walking frame

4. Walks with crutches

5. Walks with two canes

6. Walks with one cane

7. Needs leg orthosis only

8. Walks without aids

\section{Mobility for moderate distances ( 10 - 100 meters)}

0 . Requires total assistance

1. Needs electric wheelchair or partial assistance to operate manual wheelchair

2. Moves independently in manual wheelchair

3. Walks with a walking frame

4. Walks with crutches

5. Walks with two canes

6. Walks with one cane

7. Needs leg orthosis only

8. Walks without aids

\section{Mobility outdoors (more than 100 meters)}

0 . Requires total assistance

1. Needs electric wheelchair or partial assistance to operate manual wheelchair

2. Moves independently in manual wheelchair

3. Walks with a walking frame

4. Walks with crutches

5. Walks with two canes

6. Walks with one cane

7. Needs leg orthosis only

8. Walks without aids

\section{Stair management}

0 . Unable to climb or descend stairs

1. Climbs 1 or 2 steps only, in a training setup

2. Climbs and descends at least 3 steps with support or supervision of another person

3. Climbs and descends at least 3 steps with support of handrail and/or crutch and/or cane

4. Climbs and descends at least 3 steps without any support or supervision

16. Transfers: wheelchair-car (approaching car, locking wheelchair, removing arm

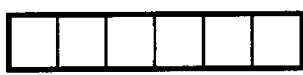
and foot rests, transferring to and from car, bringing wheelchair into and out of car)

0 . Requires total assistance

1. Needs partial assistance and/or supervision, and/or adaptive devices

2. Independent without adaptive devices 